\title{
IgA nephropathy associated with coeliac disease
}

\author{
IRENEUSZ HABURA ${ }^{1}$, KATARZYNA FIEDOROWICZI, ALDONA WOŹNIAK², \\ ILONA IDASIAK-PIECHOCKA ${ }^{3}$, PAWEŁ KOSIKOWSKI ${ }^{2}$, ANDRZEJ OKO \\ ${ }^{1}$ Department of Nephrology, Karol Marcinkowski Hospital, Zielona Góra, Poland \\ ${ }^{2}$ Department of Clinical Pathology, University of Medical Sciences, Poznań, Poland \\ ${ }^{3}$ Department of Nephrology, Transplantology and Internal Medicine, University of Medical Sciences, Poznań, Poland
}

\begin{abstract}
Coeliac disease $(C D)$ is an autoimmune disorder of the small intestine triggered by ingested gluten from barley, rye and wheat. It can be associated with other autoimmune conditions, such as type 1 diabetes, autoimmune thyroiditis and hepatitis, Sjögren's syndrome and IgA nephropathy (IgAN). We describe here a case of a 24-year-old man with the above-mentioned atypical form of coeliac disease for whom the diagnosis started with renal disorder. The diagnosis of nephrotic syndrome was established and the coexistence with CD was also suspected. In fact, immunoglobulin (Ig) A and IgG antibodies against endomysium and against gliadin were detected in serum of the patient and the endoscopic biopsy of the duodenum revealed stage $3 B C D$. Percutaneous kidney biopsy was also performed. Class I IgAN was diagnosed. Gluten-free diet, ACE inhibitor and oral iron were introduced to the patient. The improvement of clinical and laboratory disorders of $C D$ as well as gradual remission of the nephrotic syndrome were observed. In conclusion, there may be a small group of patients with IgAN coexisting with CD in whom a gluten-free diet seems to be the treatment of choice for the resolution of kidney disease.
\end{abstract}

Key words: IgA nephropathy, gluten-free diet, coeliac disease.

(Centr Eur J Immunol 2019; 44 (1): 106-108)

\section{Introduction}

Coeliac disease (CD) is an autoimmune disorder of the small intestine triggered by ingested gluten from barley, rye and wheat. The disorder occurs in genetically predisposed people - class II human leukocyte antigens (HLA), such as HLA-DQ2 or, much less commonly, HLA-DQ8, are expressed in the majority of patients with CD. It is estimated to affect 1 in 100 people worldwide. The disease has both intestinal and extraintestinal manifestations. The intestinal symptoms include abdominal pain, diarrhoea and other nonspecific abdominal symptoms. The manifestations outside the gastrointestinal tract include rashes (e.g. dermatitis herpetiformis), arthralgias, neurologic and psychiatric symptoms, fatigue, infertility, weight loss, iron deficiency anaemia, osteoporosis or osteomalacia due to calcium and vitamin D malabsorption.

Coeliac disease can be associated with other autoimmune conditions, such as type 1 diabetes, autoimmune thyroiditis and hepatitis, Sjögren's syndrome and IgA nephropathy (IgAN) [1]. Individuals with CD suffer a 3-fold increased risk of future IgAN [2] along with an increased risk of subsequent end-stage renal disease [3].
Atypical form of CD presenting with a few or even no gastrointestinal symptoms or signs is largely responsible for the increased prevalence of CD today.

\section{Case report}

We describe a case of a 24-year-old man with atypical form of $\mathrm{CD}$ for whom the diagnosis started with renal disorder.

The patient (measuring $164 \mathrm{~cm}$, BMI 20) was admitted to the Department of Internal Medicine because of peripheral oedema, headache and elevation of blood pressure $(140 / 90 \mathrm{mmHg})$. The symptoms had appeared a few days earlier. In laboratory analysis, microcytic anaemia, hypoalbuminaemia and elevation of hepatic enzymes were found. Urinary protein loss was $6 \mathrm{~g} /$ day. The diagnosis of nephrotic syndrome was established and coexistence with $\mathrm{CD}$ was also suspected. In fact, immunoglobulin (Ig) A and IgG antibodies against endomysium and against gliadin were detected in the serum of the patient and the endoscopic biopsy of the duodenum revealed markedly increased numbers of intraepithelial lymphocytes (60-70 per 100) along with crypt hyperplasia and moderate mostly lymphocytic

Correspondence: Prof. Andrzej Oko, Department of Nephrology, Transplantology and Internal Medicine, University of Medical Sciences, 49 Przybyszewskiego St., 60-355 Poznań, Poland, e-mail: aoko@ump.edu.pl

Submitted: 26.09.2016; Accepted: 08.03.2018 
A

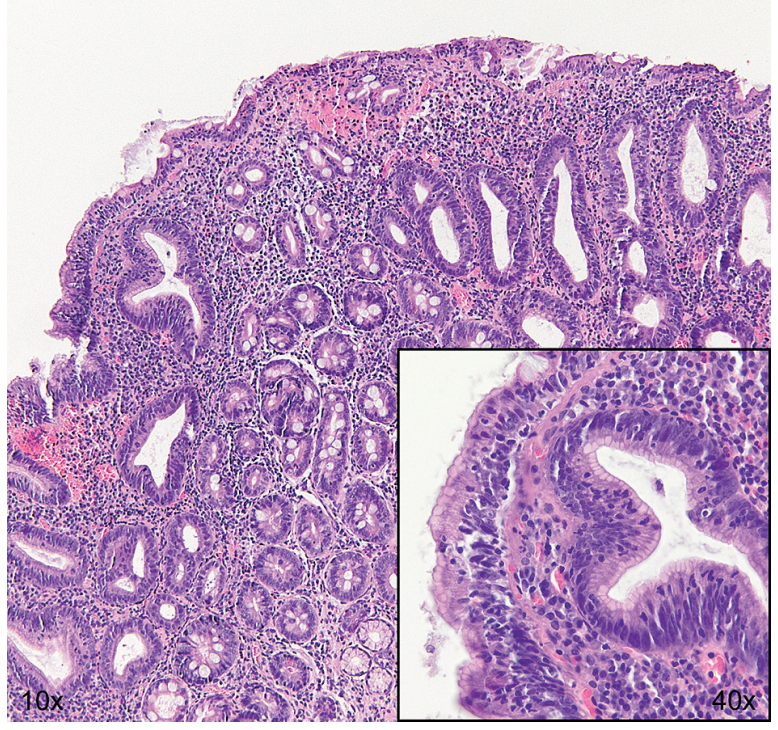

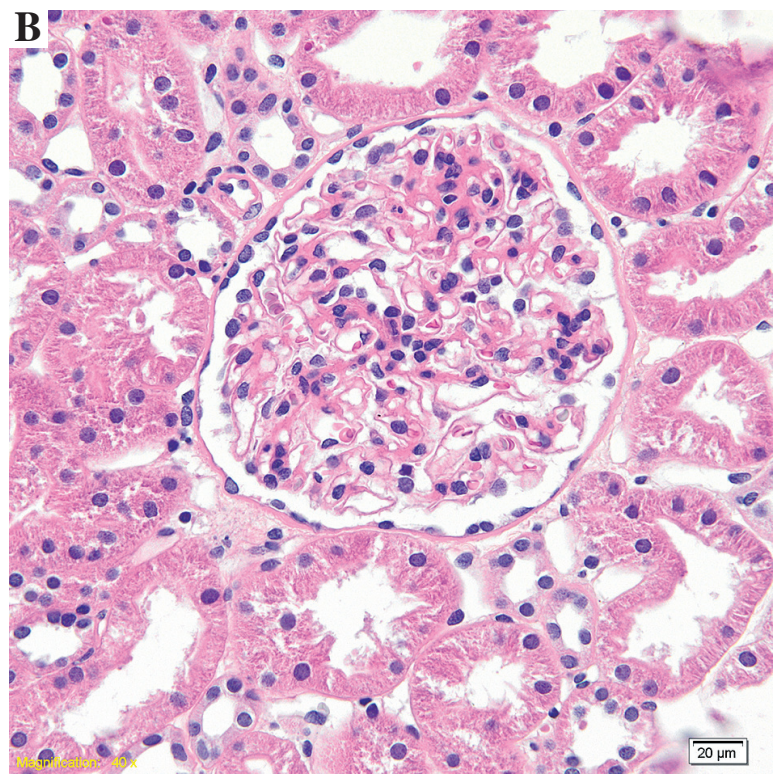

Fig. 1. Duodenal biopsy shows loss of normal villous architecture. There is extensive atrophy present with almost complete absence of villi along with marked intraepithelial lymphocytosis (insert). Moderate degree of chronic inflammation is seen in the lamina propria $(\mathrm{H} \& \mathrm{E}, 100 \times, 400 \times$ insert)

infiltrates present in the lamina propria. The villi exhibited a leaf-like appearance with moderate blunting in height (subtotal villous atrophy) (Fig. 1A). These histopathological features were classified as stage $3 \mathrm{~B}$ according to the modified Marsh-Oberhuber classification [4].

Percutaneous kidney biopsy was also performed. Immunofluorescence microscopy showed mesangial granu$\operatorname{lar} \operatorname{IgA}$ and $\mathrm{C} 3$ deposits in glomeruli. Class I IgAN with a focal increase in mesangial cells was revealed by light

Table 1. Clinical and laboratory parameters of the patient at the onset and after 6 months of therapy

\begin{tabular}{lcc}
\hline Parameters & $\begin{array}{c}\text { Before } \\
\text { treatment }\end{array}$ & $\begin{array}{c}\text { After 6 months } \\
\text { of treatment }\end{array}$ \\
\hline Body weight $(\mathrm{kg})$ & 54 & 61 \\
\hline Blood pressure $(\mathrm{mmHg})$ & $140 / 100$ & $100 / 70$ \\
\hline Haemoglobin $(\mathrm{g} / \mathrm{dl})$ & 10.1 & 14.9 \\
\hline MCV $(\mathrm{fl})$ & 79 & 92 \\
\hline Ferritin $(\mathrm{ng} / \mathrm{ml})$ & 6 & 162 \\
\hline Serum albumin $(\mathrm{g} / \mathrm{dl})$ & 2.04 & 4.4 \\
\hline AlAT $(\mathrm{U} / \mathrm{l})$ & 48 & 16 \\
\hline AspAT $(\mathrm{U} / \mathrm{l})$ & 69 & 25 \\
\hline Anti-gliadin antibodies & $\mathrm{IgA}++$, IgG++ & IgA+/-, IgG++++ \\
\hline Anti-endomysium antibodies & $\mathrm{IgA}++, \mathrm{IgG}+$ & $\mathrm{IgA}+$ \\
\hline Proteinuria (g/24 h) & 3.9 & 0.127 \\
\hline $\begin{array}{l}\text { MCV - mean corpuscular volume, AlAT - alanine aminotransferase, AspAT- } \\
\text { aspartate aminotransferase }\end{array}$ & &
\end{tabular}

microscopy (G I according to the modified H. S. Lee system); according to the Oxford classification of IgAN M0, S0, E0, T0 predictors of outcome were found [5] (Fig. 1B). Electron microscopy was not performed.

Gluten-free diet, ACE inhibitor according to KDIGO Guidelines [6] and oral iron were introduced to the patient. Gradual remission of the nephrotic syndrome was observed. Clinical and laboratory characteristics of the patient before and after 6 months of therapy are shown in Table 1.

\section{Discussion}

This case presents atypical CD associated with nephrotic syndrome due to IgAN. Two similar cases were previously described in the literature [7,8]; both presented nephrotic syndrome. Moreover in one, in contrast to our patient, renal function was markedly affected at presentation. The pathogenic link between CD and IgAN involves increased permeability of the mucosal barrier to pathogens that can stimulate the production and further deposition of pathogenic IgA in the glomerular mesangium [9]. Moreover, patients with $\mathrm{CD}$ overexpress the transferrin receptor on enterocytes, which acts as an IgA receptor, and the transferrin receptor is associated with transglutaminase 2 in CD and IgAN [10]. Recently, Kiryluk et al. [11] performed a genome-wide association study (GWAS) of IgAN and, besides nine previously reported associations, identified six new signals. Most of them were associated with either risk of inflammatory bowel disease or maintenance of the intestinal epithelial barrier, activation of mucosal $\operatorname{IgA}$ 
production and defence against intracellular pathogens. These findings could explain the genetic susceptibility of some individuals with CD to develop IgAN. On the other hand, a recent serologic study by Moeller et al. [12] did not demonstrate a significant role of CD in IgAN. In fact, in comparison with healthy subjects, there was no statistically significant increase in IgA or IgG antibody titres to gliadin in IgAN patients. Moreover, patients with IgAN and unaffected controls did not differ in the level of antibodies to deamidated gliadin and TG2, CD-specific serologic markers. A large epidemiological study from Sweden also demonstrated that only $0.026 \%$ of CD patients develop IgAN [13].

In our patient, introduction of a gluten-free diet and ACE inhibitor resulted in complete remission of nephropathy and improvement of clinical and laboratory disorders of CD. Koivuviita et al. [8] reported a case of a male patient with nephrotic syndrome and IgAN in whom the treatment with prednisone and immunosuppressive drugs was ineffective. Also in this case introduction of a gluten-free diet after the diagnosis of CD was established resulted in complete remission of kidney disease.

In conclusion, there may be a small group of patients with $\mathrm{IgAN}$ coexisting with $\mathrm{CD}$ in whom a gluten-free diet seems to be the treatment of choice for the resolution of kidney disease.

The authors declare no conflict of interest.

\section{References}

1. Riksfjord Hamnvik OP, Saldana F, Levy BD, et al. (2014): Against the Grain. N Engl J Med 371: 1333s-1338s.

2. Welender A, Sundelin B, Fored M, et al. (2013): Increased risk of IgA Nephropathy among individuals with celiac disease. J Clin Gastroenterol 47: 678-683.

3. Welender A, Prutz KG, Fored M, et al. (2012): Increased risk of end-stage renal disease in individuals with celiac disease. Gut 61: 64-68.

4. Dickson BC, Streutker CJ, Chetty R (2006): Coeliac disease: an update for pathologists. J Clin Pathol 59: 1008-1016.

5. Lee HS, Lee MS, Lee SM (2005): Histological grading of Ig A nephropathy predicting renal outcome: revisiting H. S. Lee's glomerular grading system. Nephrol Dial Transplant 20: 342-348.

6. KDIGO Clinical Practice Guidelines for Glomerulonephritis (2012); 2: 209-217.

7. Woodrow G, Innes A, Boyd SM, Burden RP (1993): A case of IgA nephropathy with coeliac disease responding to a gluten-free diet. Nephrol Dial Transplant 8: 1382-1383.

8. Koivuviita N, Tertti R, Heiro M, Metsarinne K (2009): A case report: a patient with IgA nephropathy and coeliac disease. Complete clinical remission following gluten-free diet. Nephrol Dial Transplant 2: 161-163.

9. Rantala I, Collin P, Holm K et al. (1999): Small bowel T cells, HLA class II antigen DR, and GroEL stress protein in IgA nephropathy. Kidney Int 55: 2274-2280.
10. Floege J, Feehally J (2016): The mucosa-kidney axis in IgA nephropathy. Nat Rev Nephrol 12: 147-156.

11. Kiryluk K, Li Y, Scolari F, et al. (2014): Discovery of new risk loci for IgA nephropathy implicates genes involved in immunity against intestinal pathogens. Nat Genet 46: 1187-1196.

12. Moeller S, Canetta PA, Taylor AK, et al. (2014): Lack of serologic evidence to link IgA nephropathy with celiac disease or immune reactivity to gluten. PloS One 9: e94677.

13. Welander A, Sundelin B, Fored M, Ludvigsson JF (2013): Increased risk of IgA nephropathy among individuals with celiac disease. J Clin Gastroenterol 47: 678-683. 